\title{
Implementation and Evaluation of a Team-Based Approach to Hospital Discharge Transition of Care
}

Matthew Van De Graaf, BS | Hemal Patel, MD | Brynn Sheehan, PhD | Jennifer Ryal, MD PRiMER. 2021;5:28.

Published: 8/27/2021 | DOI: 10.22454/PRiMER.2021.675929

\section{Abstract}

Background: Transitional care management (TCM) programs guide patients from hospital discharge to outpatient follow-up with the goal to decrease hospital readmissions and the cost of care. In 2017, the department of primary care internal medicine (PCIM) at Eastern Virginia Medical Group implemented TCM. We aimed to evaluate the efficacy and self-sustainability of this TCM program.

Methods: The TCM team contacted patients upon discharge to schedule the follow-up appointment. We coded patient contact as (1) no successful phone-call contact, patient did not attend appointment; (2) successful phone-call contact, patient did not attend appointment; and (3) patient attended appointment. We collected patient demographics, readmissions, and visit costs using manual chart review and electronic health record (EHR) data extraction. We conducted $\chi^{2}$ analysis, one-way analysis of variance, and unpaired $t$ tests to assess associations between readmission rates or costs and TCM care.

Results: Initial analysis did not indicate significant associations between readmission rates and level of TCM care at $30\left(\chi^{2}=1.40, P=.50\right), 60\left(\chi^{2}=5.48, P=.06\right)$, or $90\left(\chi^{2}=4.23, P=.12\right)$ days or significant differences in patient charges at $30(F[2,59]=2.85, P=.06), 60(F[2,91]=2.00, P=.14)$, or $90(F[2,126]=1.39, P=.25)$ days. Follow-up analysis indicated significant associations between readmission rates and any level of TCM care at $60\left(\chi^{2}=5.40, P=.02\right)$ and $90\left(\chi^{2}=4.21, P=.04\right)$ days, but not at 30 days $\left(\chi^{2}=1.39, P=.28\right)$.

Conclusions: Our TCM program review suggests that the benefits of transitional care extend beyond 30 days by decreasing readmission rates at 60 and 90 days after hospital discharge.

\section{Introduction}

Transitional care management (TCM) is a robust intervention to guide a patient's transition from a hospital setting to an outpatient follow-up visit with a primary care physician. ${ }^{1}$ Adherence to treatment plans can be low, ${ }^{2}$ and nonadherence to discharge instructions is associated with poor health outcomes. ${ }^{3,4}$ In January 2013, the Center for Medicare and Medicaid Services (CMS) created new billing codes $(99495,99496)$ to address the work involved in coordinating postdischarge services, ${ }^{5}$ incentivizing TCM programs by increasing reimbursements to physicians who provide transitional care. ${ }^{6}$ Comprehensive discharge planning and follow-up has been shown to reduce hospital readmissions 30 days postdischarge ${ }^{7-9}$ and the benefit of these programs extends to physicians and their practices. ${ }^{10}$ The benefit of transitional care includes reduced future readmissions, a reduction in medication complications, and increase in high-value care for the patient. Some 
studies suggest that the benefits of transitional care extend beyond 30-days after hospital discharge. ${ }^{11}$ Here, we outline the implementation of a TCM program and report hospital readmission rates and costs to the patient.

\section{Methods}

In November 2017, the department of primary care internal medicine (PCIM) at Eastern Virginia Medical Group implemented a TCM program. A designated licensed practical nurse dedicates 0.5 full-time equivalent (FTE) to the TCM program. The TCM nurse tracks PCIM patients admitted to hospital systems and calls patients within 48 hours of hospital discharge to (1) confirm hospital discharge, (2) reconcile medication lists, (3) ensure patients fill any newly prescribed medications and stop discontinued medications, and (4) schedule follow-up appointments. There were instances in which the TCM nurse was unable to contact a patient, but the patient already had an appointment scheduled by the inpatient team. The TCM nurse attempted to schedule all appointments within 7 days of discharge but made appointments within a later time frame when requested by the patient. We included appointments following hospital discharge in our study regardless of whether they were billed as a TCM visit.

From November 2017 to March 2019, we tracked all-cause hospital admissions for PCIM patients at a local hospital system. The cohort included patients who were discharged home or to another care facility. We performed manual chart review to collect patient data on all-cause hospital readmissions after discharge and received electronic health record (EHR) cost data associated with patient readmissions at 30,60, and 90-day intervals from the initial date of discharge. Additionally, we recorded patient age, length of hospital stay, and number of problems treated during hospitalization. To assess differences in outcomes (eg, readmission rates, readmission charges) associated with TCM care, we conducted $\chi^{2}$ analysis, one-way analysis of variance (ANOVA), and unpaired $t$ tests. The Eastern Virginia Medical School Institutional Review Board approved this study.

\section{Results}

There were 574 patients included in the study with an average age of $64.1( \pm 14.4)$ years. The patients had an average hospital length of stay of $4.0( \pm 3.6)$ days, and an average of $6.6( \pm 4.6)$ problems treated during hospitalization. To evaluate the program, we divided patients into three groups: (1) patients who did not receive any transitional care $(n=99,17.2 \%),(2)$ patients who received a phone call but did not attend an appointment $(n=122,21.3 \%)$, and (3) patients who attended a transitional care appointment ( $n=353,61.5 \%$; Figure 1$)$.

When evaluating all-cause hospital readmissions, patients who received no transitional care had numerically higher readmission rates compared to those who received a phone call or attended an appointment (Figure 2). However, $\chi^{2}$ analyses did not indicate statistically significant associations between readmission rates and level of TCM care completed at $30\left(\chi^{2}=1.40, P=.50\right), 60\left(\chi^{2}=5.48, P=.06\right)$, or $90\left(\chi^{2}=4.23, P=.12\right)$ days. Likelihood ratios associated with each chi-square analysis were $1.31(P=.52), 5.05(P=.08)$, and $4.00(P=.14)$ for 30,60, and 90-day readmissions, respectively. Similarly, one-way ANOVAs assessing differences in readmission charges (Figure 3) did not demonstrate statistically significant differences at $30(F[2,59]=2.85, P=.06), 60(F[2,91]=2.00$, $P=.14)$, or $90(F[2,126]=1.39, P=.25)$ days.

To assess whether any TCM intervention provides a benefit when compared with no TCM care, we considered the TCM appointment and TCM call categories jointly. $\chi^{2}$ analyses indicated significant associations between readmission rates and any level of TCM care at $60\left(\chi^{2}=5.40, P=.02\right)$ and $90\left(\chi^{2}=4.21, P=.04\right)$ days, but not at 30 days $\left(\chi^{2}=1.39, P=.28\right)$. Likelihood ratios associated with each $\chi^{2}$ analysis were $1.30(P=.25), 4.96(P=.02)$, and $3.98(P=.04)$ for 30,60 , and 90 day readmissions, respectively. Unpaired $t$-test analysis of readmission cost 
data with the TCM appointment and TCM call categories considered jointly indicated that results were not significant at $30(P=.73), 60(P=.07)$, or $90(P=.41)$ days.

\section{Conclusions}

We outline a clinically relevant intervention for transitional care management, but our study does have limitations. The small number of readmitted patients likely limited power to detect a statistically significant effect in costs. Additionally, we were only able to analyze readmissions and cost data available through one hospital system. Although this system includes multiple hospitals, any admissions outside of the hospital system were not included in the analysis. Moreover, our analysis does not stratify patients by age, length of hospital stay, risk for readmission, or other factors. While our data are insufficient to draw conclusions about the financial impacts of TCM, implementation of transitional care programs likely has implications for revenue at the primary care practice and health system level.

Our data build upon previous studies by indicating the strength of scheduling follow-up appointments through multiple processes, as $39.6 \%$ of patients who were not contacted by the TCM nurse attended an appointment due to the work of in-patient teams scheduling follow-up. While the capacity for transitional care programs to improve patient care has been well established in previous studies, ${ }^{7-9,11}$ we demonstrate that the benefit of decreased readmissions may extend beyond the 30-day time interval, as analysis indicates that any form of TCM care is associated with reductions in readmission rates at 60 and 90 days. Despite their limitations, our findings emphasize the positive impact of transitional care and provide a framework for practices seeking to implement and evaluate TCM programs.

\section{Tables and Figures}

Figure 1: Workflow of Our Transitional Care Management Program

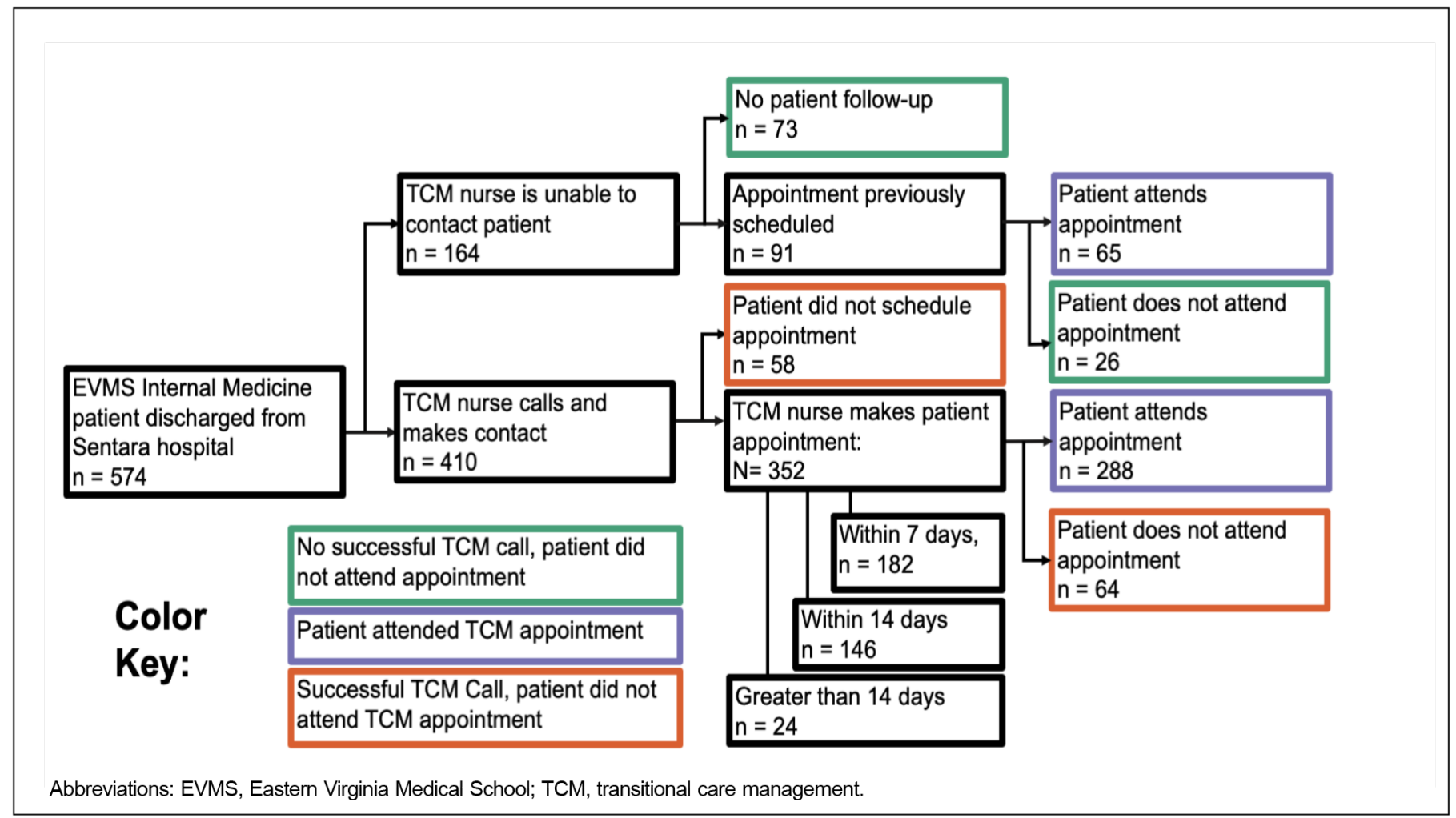


Figure 2: All-Cause Hospital Readmissions by TCM Level

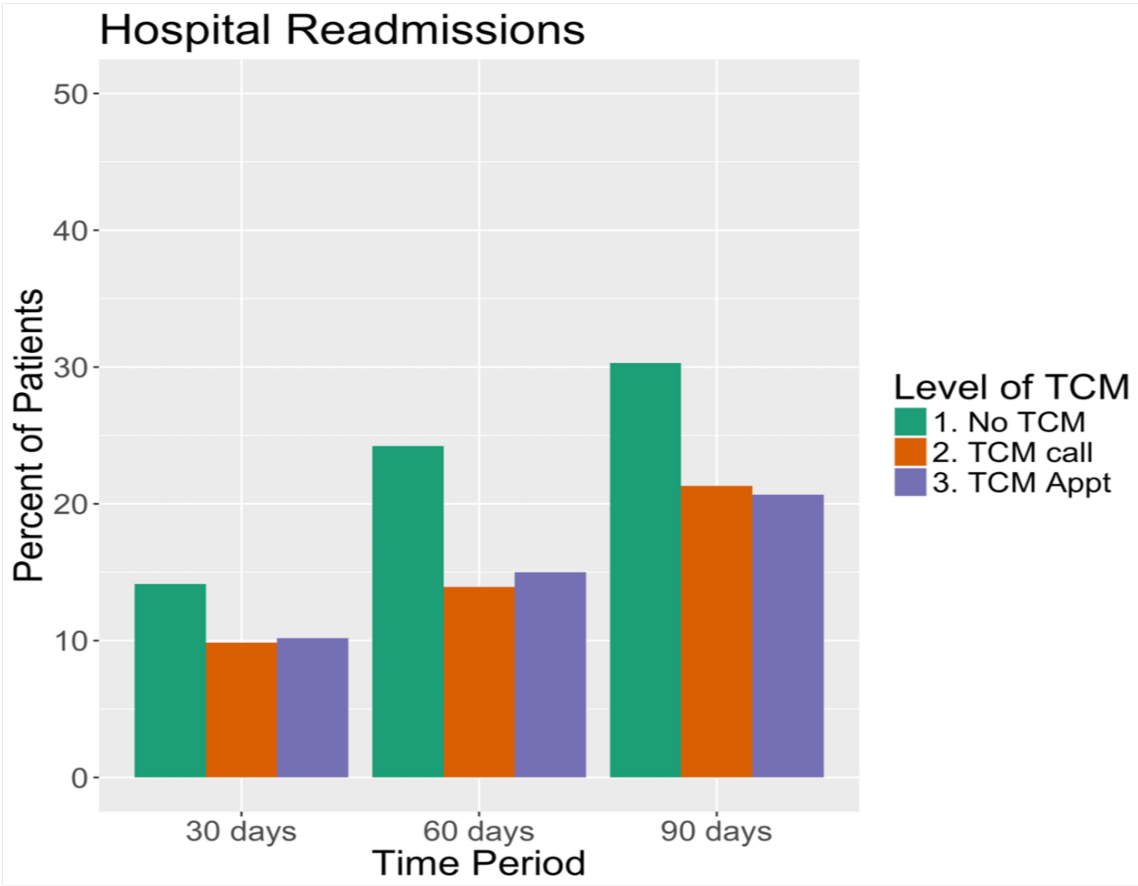

Abbreviation: TCM, transitional care management.

Figure 3: Patient Charges by TCM Level

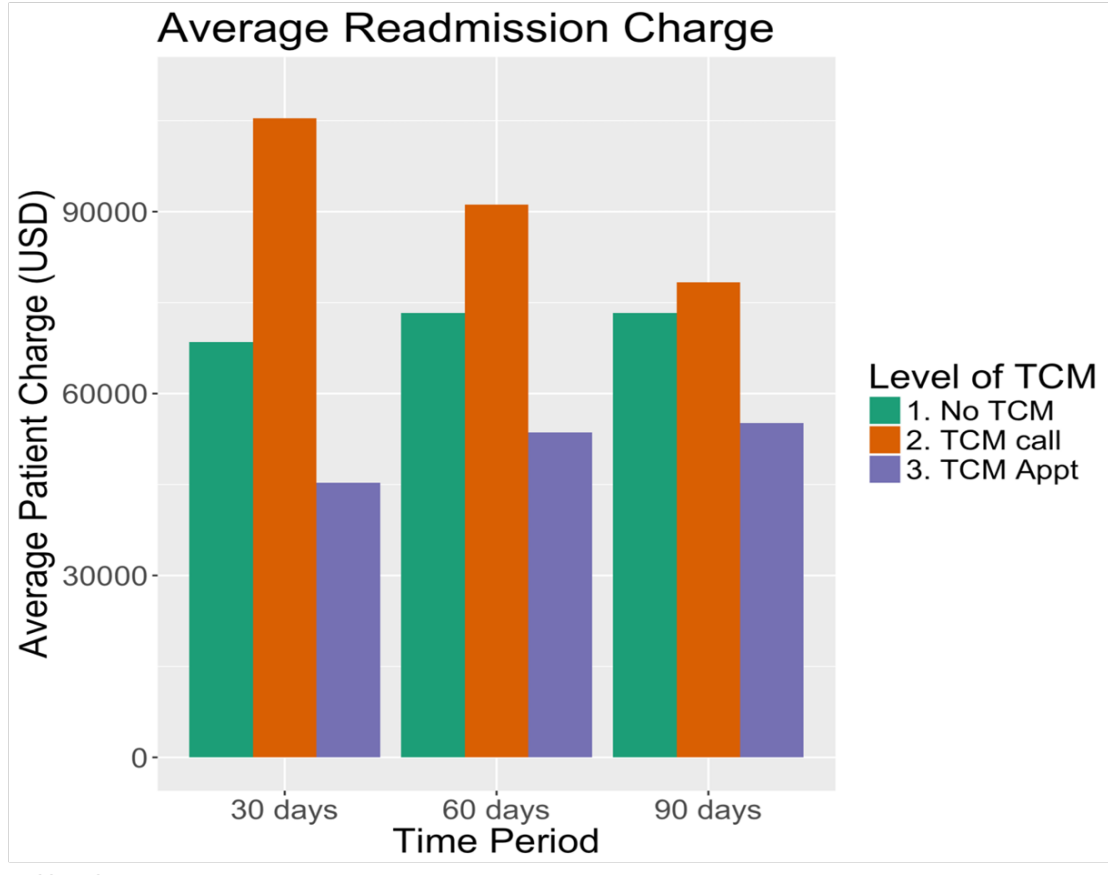

Abbreviation: TCM, transitional care management.

\section{Acknowledgments}

Presentations: This study was presented at the following venues:

American College of Physicians, Virginia Chapter Annual Meeting (virtual). March 2020. 
December 2019: STFM 2019 Conference on Practice and Quality Improvement. Phoenix, AZ. December, 2019.

\section{Corresponding Author}

Jennifer Ryal, MD

Eastern Virginia Medical School: Department of Internal Medicine, EVMS Internal Medicine, 825 Fairfax Ave, Norfolk, VA 23507. 757-446-8910. Fax: 757-446-7114.

ryaljl@evms.edu

\section{Author Affiliations}

Matthew Van De Graaf, BS - Eastern Virginia Medical School, Norfolk, VA

Hemal Patel, MD - Eastern Virginia Medical School, Department of Geriatrics, Norfolk, VA

Brynn Sheehan, PhD - Eastern Virginia Medical School, Department of Psychiatry and Behavioral Sciences | and Healthcare Analytics and Delivery Science Institute, Norfolk, VA

Jennifer Ryal, MD - Eastern Virginia Medical School, Department of Internal Medicine, Norfolk, VA

\section{References}

1. Patel NK, Mathew R, Aniemeke C, Tripathy C, Jaen CR, Tysinger J. Transitional care management: practical processes for your practice. Fam Pract Manag. 2019;26(3):27-30.

2. Osterberg L, Blaschke T. Adherence to medication. N Engl J Med. 2005;353(5):487-497. doi:10.1056/NEJMra050100

3. Forster AJ, Murff HJ, Peterson JF, Gandhi TK, Bates DW. The incidence and severity of adverse events affecting patients after discharge from the hospital. Ann Intern Med. 2003;138(3):161-167. doi:10.7326/0003-4819-138-3-200302040-00007

4. Forster AJ, Murff HJ, Peterson JF, Gandhi TK, Bates DW. The incidence and severity of adverse events affecting patients after discharge from the hospital. Ann Intern Med. 2003;138(3):161-167. doi:10.7326/0003-4819-138-3-200302040-00007

5. Bloink J, Adler KG. Transitional care management services: new codes, new requirements. Fam Pract Manag. 2013;20(3):12-17.

6. Adler KG. Transitional care management: why bother? Fam Pract Manag. 2013;20(3):6-6.

7. Hitch B, Parlier AB, Reed L, Galvin SL, Fagan EB, Wilson CG. Evaluation of a team-based, transition-of-care management service on 30-day readmission rates. N C Med J. 2016;77(2):87-92.

doi:10.18043/ncm.77.2.87

8. Misky GJ, Wald HL, Coleman EA, Misky GJ. Post-hospitalization transitions: examining the effects of timing of primary care provider follow-up. J Hosp Med. 2010;5(7):392-397. doi:10.1002/jhm.666

9. Mansukhani RP, Bridgeman MB, Candelario D, Eckert LJ. Exploring transitional care: evidence-based strategies for improving provider communication and reducing readmissions. P\&T. 2015;40(10):690-694.

10. Adler KG. Transitional care management: why bother? Fam Pract Manag. 2013;20(3):6-6.

11. Bindman $A B$, Cox DF. Changes in health care costs and mortality associated with transitional care management services after a discharge among Medicare beneficiaries. JAMA Intern Med. 2018;178(9):1165-1171. doi:10.1001/jamainternmed.2018.2572

Copyright $\odot 2021$ by the Society of Teachers of Family Medicine 